\title{
DEL DISCURSO A LA LEXICOGRA- FÍA: PROPUESTA DE APLICACIÓN PARA LOS CONECTORES REFORMULATIVOS
}

\author{
Carme Bach Martorell
}

\begin{abstract}
RESUMO: This paper aims at defending the necessity of creating applications from studies on discourse, so that applied linguistics becomes a useful tool for the society. This work explains briefly what reformulation connectors are and it shows the electronic prototype (than can be consulted by internet) of a tool designed to make the work of lexicographers easy concerning the entrance of connectors in the dictionaries (ALCOR), by making emphasis on the theoretical base on which it is sustained and on the decisions of application that have been taken into account.
\end{abstract}

PALAVRAS-CHAVE: conector, reformulação, aplicação ao discurso, lexicografia, dicionário de partículas.

Por una parte, nuestro interés inicial por los conectores reformulativos y la consideración que los trabajos de investigación deben desembocar necesariamente en la construcción de recursos directamente

Carme Bach Martorell é pesquisadora do Grupo Iulaterm, Institut Universitari de Lingüística Aplicada, Universidat Pompeu Fabra, Espanha. 
relacionados con aplicaciones lingüísticas, nos conduce a observar que existe un gran distanciamiento entre los estudios sobre el discurso de orientación teórico-descriptiva y los de orientación aplicada.

Por otra parte, la observación preliminar de las carencias y asistematicidades de las obras lexicográficas en relación con el tratamiento de los conectores reformulativos refuerza la conveniencia de desarrollar recursos aplicados que faciliten su tratamiento sistemático en los diccionarios. Además, la constatación que es posible establecer unos valores mínimos estables para cada conector, a pesar de la aparente polisemia de los conectores, permite plantearnos el diseño de un recurso aplicado que ayude al lexicógrafo a mejorar la representación de estas unidades en los diccionarios.

En este artículo explicamos brevemente qué entendemos por conectores reformulativos y describimos la aplicación resultante de un estudio teórico-descriptivo sobre estos conectores ${ }^{1}$, explicando la base teórica en la que se fundamenta y las decisiones de diseño que hemos tomado.

\section{LOS CONECTORES REFORMULATIVOS}

El estudio de los conectores reformulativos se lleva a cabo en el marco de los estudios lingüísticos sobre análisis del discurso. En concreto, nuestro análisis se sitúa en una propuesta semántico-pragmática composicional, en la que se consideran aspectos de tres marcos teóricos pragmático-discursivos complementarios: la Teoría de la argumentación (Ducrot 1980, Anscombre 1995 y Ducrot \& Anscombre 1983); la Escuela clásica de Ginebra (Roulet 1987) y por último, la Nueva escuela integradora ginebrina, que considera aspectos de los dos marcos teóricos anteriores juntamente con elementos de la Teoría de la relevancia de Sperber y Wilson (1986), cuyo máximo exponente es la obra de Moeschler J. et. al. (1994). Centrándonos en el análisis de los conectores reformulativos, los trabajos de Gülich \& Kotschi $(1983,1987,1995)$ y Fuchs $(1982,1994 a)$ han supuesto el punto de partida de nuestro trabajo.

Una vez presentada la propuesta teórica mediante la cual se ha hecho el análisis de las unidades léxicas reformulativas, pasamos a presentar el concepto de conector reformulativo del que partimos.

Los conectores reformulativos son unidades léxicas, formadas por uno o más morfemas, especializadas en la reformulación textual, que vehiculan la actividad metadiscursiva y, a su vez, facilitan la interacción comunicativa como organizadores textuales. Las piezas léxicas que nos

${ }^{1}$ Para más detalles puede consultarse la tesis doctoral Bach, C. (2001), dirigida por los doctores M. Teresa Cabré y L. Payrató.

302 
ocupan actúan como conectores en la medida en que enlazan dos enunciados del discurso indicando a su vez la relación semántico-pragmática que se establece entre ellos, de forma dinámica: és a dir 'es decir', més ben dit 'más bien dicho', dit d'una altra manera 'dicho de otro modo', o sigui 'o sea', de fet 'de hecho' y de tota manera 'de todos modos' son algunos de los conectores reformulativos sobre los cuales hemos centrado nuestra atención.

Dentro de los conectores reformulativos podemos establecer dos subgrupos claramente diferenciados: los reformulativos parafrásticos y los no parafrásticos, que se diferencian tanto por el tipo de conector que vehicula las operaciones de reformulación en cada caso, como por la capacidad de establecer equivalencia o distanciamiento entre los enunciados conectados (reformulativos parafrásticos / reformulativos no parafrásticos).

Los pocos trabajos existentes que plantean algún tipo de descripción lexicográfica para las unidades conectivas destacan su polisemia. De todos modos, Paillard (1991) apunta que, bajo la aparente dispersión funcional del los conectores, es posible encontrar una caracterización general de las unidades, válida para todas sus ocurrencias, aunque después sea necesario precisar sus "valores locales".

En relación con las indicaciones de Paillard, el análisis realizado ha permitido constatar que, bajo la aparente polisemia de los conectores reformulativos, se encuentra un valor semántico de base para cada unidad y una serie de valores particulares que se actualizan en función del contexto en que aparecen, directamente relacionados con las instrucciones de base y de segundo nivel postuladas por Luscher (1989) dentro del marco teórico del que partimos. De este modo, consideramos que la polisemia en las unidades léxicas que nos ocupan es tan sólo aparente y que es posible realizar una única entrada lexicográfica para cada conector reformulativo como se observa en la aplicación que describimos.

\section{BASE TEÓRICA DE LAAPLICACIÓN LEXICOGRÁFICA}

En primer lugar, debemos señalar que no concebimos una aplicación lingüística como la que aquí proponemos si no que se apoya en una propuesta teórica (CABRÉ, 1990, p. 10).

Por este motivo, relacionamos nuestra propuesta de aplicación lexicográfica con la Teoría del continuo semántico, representada fundamentalmente en las obras de Fuchs (1994b, 1997), Victorri (1996) y Fuchs \& Victorri $(1992,1994)$. Esta propuesta teórica permite explicar la aparente polisemia de los conectores reformulativos como una gradación semántica en la cual aparecen unos elementos mínimos estables, necesarios 
para una formalización computacional o lexicográfica como la que nos planteamos. Según la propuesta de Victorri y Fuchs, el resto de rasgos de las unidades léxicas que ellos denominan 'inestables' se genera dinámicamente en función del contexto en que aparecen:

"Le noyau de sens ainsi défini présente donc un double aspect. D'une part, sur un plan strictement linguistique, il permet d'étudier le fonctionnement sémantique de l'expression en separant nettement la partie invariable de son sémantisme, ce qu'il apport à la signification de tout énoncé qui le contient, de la partie variable qui est determinée par l'interaction avec les expressions co-présentes dans l'énoncé. C'est cet aspect qui nous intéresse ici. Mais d'autre part, cette définition en tant qu'opérateur abstrait ouvre la voie à une représentation cognitive de cette opération." (FUCHS y VICTORRI, 1992, p. 143-144)

Aunque compartimos estas nociones teóricas, debemos indicar que no consideramos que el significado se genere dinámicamente sino que creemos que en función del contexto en que aparece cada conector se produce la actualización dinámica de unos valores u otros. Hacemos esta consideración teórica porque creemos que el uso del término generación podría llevar implícita la idea errónea de que la actualización de significados asociados a cada unidad podría ser ilimitado, hecho que contradice el análisis de los conectores reformulativos realizado.

Una vez presentado el marco teórico en el que se inscribe nuestra propuesta, detallamos las decisiones de aplicación de las cuales partimos.

\section{DECISIONES DE APLICACIÓN}

En primer lugar, debemos señalar que, considerando las posibilidades que la informática ofrece en la actualidad, hemos optado por la realización de un prototipo lexicográfico de tipo electrónico, consultable a través de internet, ya que permite mucha más elasticidad en la implementación de una aplicación como la que nos hemos propuesto.

La aplicación que presentamos, que denominamos ALCOR (Aplicación Lexicográfica para los Conectores Reformulativos) pretende ser un prototipo útil para los lexicógrafos en la construcción de los diccionarios y no una aplicación cerrada y completa.

ALCOR se ha concebido como una aplicación hipertextual y dinámica que no se limita únicamente a la selección de páginas $\mathrm{html}$ previamente construidas sino que el usuario interactúa con la aplicación de modo que las páginas de entrada cambian de comportamiento en función de la interacción con el usuario (el lexicógrafo en nuestro caso). 
En ALCOR, los datos se implementan una sola vez y el lexicógrafo que lo utiliza es quien decide qué unidades quiere describir (selección), qué información quiere dar para cada conector y cómo la quiere representar, en función de los objetivos y de los criterios del diccionario que esté confeccionando.

\section{PUNTOS DE ACCESO A LA INFORMACIÓN}

ALCOR presenta tres accesos a la información: la entrada, la base de referencia y el valor proposicional, accesibles desde cualquiera de las pantallas del programa.

a) Entrada

En el acceso por entrada aparece una lista de formas que el lexicógrafo puede seleccionar para consultar la información que se les asocia. Los ítems seleccionables desde esta opción pueden ser entradas con un artículo propio en el diccionario, en función del criterio de lematización que se aplique a la obra lexicográfica en constitución.

b) Base de referencia

Cuando el criterio que se aplica en el diccionario con que trabaja el lexicógrafo indica que los conectores deben describirse bajo el núcleo de la palabra semánticamente plena sobre la que están formados, ALCOR ofrece una lista de núcleos lemáticos a seleccionar. Una vez se ha hecho la selección, se despliega una lista que presenta todos los conectores que deberían describirse bajo el lema escogido, con la intención que el lexicógrafo disponga de una lista exhaustiva que le permita decidir qué conectores quiere incluir en su diccionario bajo aquel lema. Supongamos que el lexicógrafo quiere saber qué conectores podrían describirse bajo el lema decir. A partir de la propuesta de ALCOR, la búsqueda por el núcleo lemático decir, remite a tres conectores reformulativos és a dir (es decir) dit d'una altra manera / dit d'altra manera (dicho de otro modo) y més ben dit / millor dit (mejor dicho).

c) Valor proposicional

El acceso por el valor proposicional difiere un poco del que se hace por la entrada o la base de referencia.

Desde la opción "valor proposicional" se pueden seleccionar un total de dieciséis términos que corresponden a las funciones de las unidades discursivas que se describen a través de ALCOR: aclaración, argu- 
mentación, argumentación orientada, contraargumentación, conclusión, continuación del hilo discursivo, corrección, denominación, designación, distanciamiento, enumeración, equivalencia, ejemplificación, operación matemática, recapitulación y reformulación.

A través de la selección de las opciones que se presentan, se accede a una pantalla en la que se define la función discursiva escogida y se ofrece la posibilidad de consultar algunos ejemplos reales de los diferentes conectores que vehiculan el valor lógico-semántico en cuestión, que pertenencen al corpus utilizado para el estudio teórico-discursivo previo.

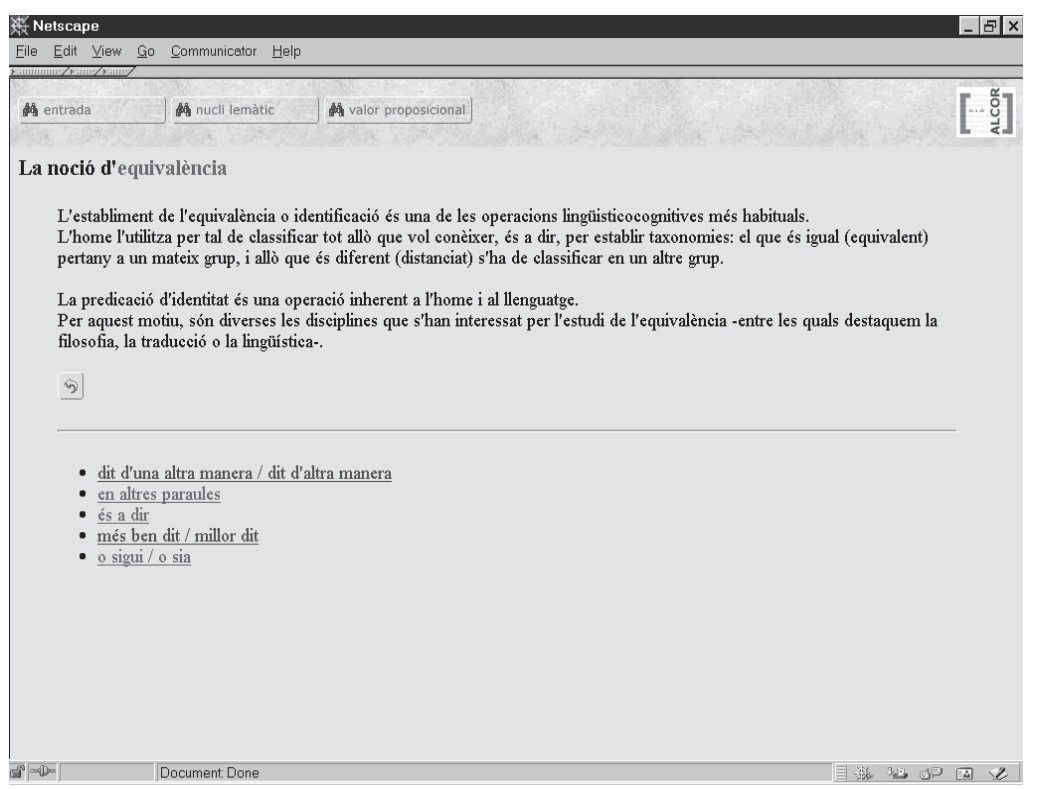

Fig. 1

Figura 1: Información para el valor proposicional «equivalencia" con los enlaces a los conectores que en todas sus ocurrencias vehiculan dicho valor.

\section{INFORMACIÓN PARA CADA CONECTOR Y REPRESENTACIÓN}

Cuando el usuario de ALCOR selecciona un conector desde cualquiera de los accesos posibles, se inicia la consulta de la información asociada a cada unidad, o dicho de otra forma, la consulta de lo que denominamos la ficha lexicográfica.

La ficha lexicográfica de ALCOR presenta dos formatos diferen- 
tes: el formato de acceso y el formato discursivo, nuevamente con la intención de facilitar el trabajo al lexicógrafo. Las dos fichas comparten la misma información pero la forma de presentar los datos y la manera de recuperar la información en vistas a su explotación son diferentes.

En primer lugar, en la cabecera de la ficha se presenta la entrada, es decir, el conector. Cuando la unidad léxica consultada presenta variaciones formales, ALCOR da en la entrada todas las variantes posibles del conector. En negrita se indica la parte del conector que nunca varía y en verde, las partes cambiables.

En segundo lugar, ALCOR presenta la información categorial que corresponde a las unidades que describe. Es importante señalar que consideramos necesario el etiquetado uniforme de les unidades conectoras. Con esta finalidad ALCOR propone un conjunto de tres etiquetas para los conectores reformulativos, representadas también gráficamente como tales:

- connector , (conector) válida para la implementación de cualquier conector en ALCOR,

- reformulatiu, (reformulativo) para la implementación de cualquier unidad reformuladora y,

- parafràstic o no parafràstic (parafrástico o no parafrástico) en función de la vehiculación de la equivalencia o del distanciamiento por parte de la unidad léxica que se describe.

Somos conscientes que la categorización como conectores de las unidades que nos ocupan puede plantear problemas al lexicógrafo por el hecho que las unidades léxicas que pretende describir ALCOR engloban unidades con características morfosintácticas diferentes. De todos modos, creemos que el hecho de compartir todas ellas unas mismas instrucciones funcionales favorece el marcaje homogéneo de estas unidades.

En tercer lugar, se describe la unidad léxica seleccionada. Como ya hemos señalado, la única definición lexicográfica posible para los conectores es la que presenta sus características funcionales es decir, la que se hace en metalengua del signo.

Con esta intención ALCOR presenta los rasgos funcionales de los conectores que se han implementado gráficamente:

a) en negrita se marcan las características funcionales compartidas por todas las ocurrencias de un mismo conector. En nuestra opinión esta es la mínima información que debería incluirse en la definición lexicográfica de los conectores porque corresponde a su núcleo funcional. 
b) indexados bajo la definición básica del conector, se presentan el resto de los valores semántico-pragmáticos que se actualizan de maneras diferentes en interacción con el contexto, así como ejemplos de cada actualización, a los que se accede haciendo un clic sobre la nota que aparece al lado de cada rasgo particular. Estos valores alternativos forman parte de la periferia funcional del conector. Sería interesante que el lexicógrafo pudiera dar cuenta de estos valores secundarios en el diccionario para dar una definición completa y exhaustiva del conector.

c) en algunos casos, la ficha lexicográfica presenta informaciones complementarias referentes al funcionamiento pragmático del conector que se describe y que el lexicógrafo puede incluir optativamente en la definición del conector.

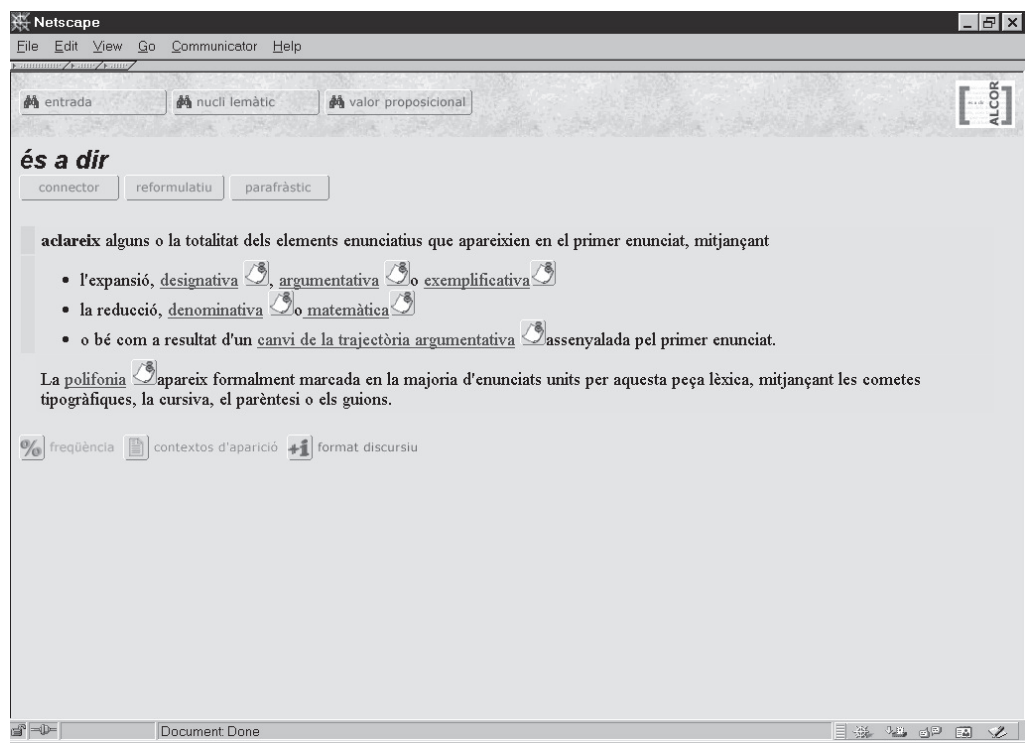

Fig. 2

Figura 2: Ficha lexicográfica para la entrada és a dir (es decir)

Finalmente como se observa en la figura anterior, en la parte inferior de la ficha, se accede a tres botones distintos:

- $\%$ frequèncla

- II contextos d'aparició y,

- $\quad+\mathbf{i}$ format discursiu 
El botón de freqüència tiene en cuenta la importancia que puede tener para el lexicográfico la frecuencia de aparición del conector en un corpus de referencia que podría consultar para confeccionar el diccionario. ${ }^{2}$ De este modo, será el lexicógrafo quien establecerá cuál es la mínima frecuencia necesaria de aparición de una unidad en el corpus de referencia para que ésta sea descrita.

El botón contextos d'apararició se incluye por la conveniencia de la ejemplificación de los distintos usos de los conectores en los diccionarios y de la anotación del comportamiento sintáctico-discursivo de estas unidades. ALCOR facilita el acceso a estas informaciones a través de este icono. Si se hace un clic sobre éste se despliega una pantalla que presenta la información de aparición contextual del conector.

Finalmente, a través del botón format discursiu se accede a un formato distinto de presentación de la información lexicográfica.

Por último, cabe destacar que la recuperación de los datos proporcionados por ALCOR se hace a partir de los mecanismos estándar de traspaso de la información que prevé el sistema operativo Windows.

Para concluir este artículo, queremos señalar que en nuestra opinión, el prototipo lexicográfico que hemos diseñado puede ser útil también para la descripción de otros conectores y que creemos que es posible e interesante diseñar un diccionario para conectores a partir de la propuesta que hemos presentado. Consideramos que ALCOR puede ser también de utilidad para la enseñanza de lengua a adultos y extranjeros y para la traducción, convenientemente complementada con información específica en cada caso.

\section{BIBLIOGRAFÍA}

ANSCOMBRE, J. C.et al. Théorie des topoï. París, Kimé, 1995.

BACH, C. Els connectors reformulatis catalans: Anàlisi i proposta d'aplicació lexicogràfica. Barcelona, Universitat Pompeu Fabra, 2001, consultable a través de internet en la dirección http://www.tdcat.cesca.es

CABRÉ, M. T. «Presentació». En: CABRÉ, M. T et al. La lingüística aplicada. Noves perspectives, noves professions, noves orientacions.Barcelona, Fundació Caixa de Pensions i Publicacions de la Universitat de Barcelona, 1990. p. 9-10.

DUCROT, O. Les mots du discours. París, Minuit, 1980.

\footnotetext{
${ }^{1}$ De este modo, en función del tipo de obra lexicográfica que se esté confeccionando se partirá de un corpus de referencia u otro.
} 
DUCROT, O. y ANSCOMBRE, J. C. (1983) La argumentación en la lengua. Madrid, Gredos, 1994. Traducción de Marta Tordesillas.

FUCHS, C. La Paraphrase. París, Presses Universitaires de France, 1982. Paraphrase et énonciation. París, Ophrsys, 1994a.

. «The challenges of continuity for a linguistic approach to semantics». En: FUCHS, C. y B. VICTORRI. (ed.) Continuity in linguistic semantics. Linguisticae Investigationes Suplementa, 19. Amsterdam, John Benjamins Publishing Co, 1994b. p. 93-107.

. "L'interprétation des polysèmes grammaticaux en contexte". En: KLEIBER, G. y M. RIEGEL (ed.) Les formes du sens. Études de linguistique française, médiévale et générale offertes à Robert Martin à l'occasion des ses 60 ans. Louvain-la Neuve, Duculot, 1997. p. 127133.

FUCHS, C. y VICTORRI, B. «Construire un espace sémantique pour représenter la polysémie d'un marqueur grammatical: L'exemple de encore». Linguisticae investigationes XVI /1, 1992. p. 125-153.

. (ed.) Continuity in linguistic semantics. Linguisticae Investigationes Suplementa, 19. Amsterdam, John Benjamins Publishing Co, 1994.

GÜLICH, E. y KOTSCHI, T. «Les marqueurs de reformulation paraphrastique». Cahiers de Linguistique Française 5, 1983. p. 305-351. . «Les actes de reformulation dans la consultation La dame de Caluire». En: BANGE, P. (ed.) L'analyse des interactions verbales. La dame de Caluire: une consultation. Actes du colloque tenu a l'Université Lyon 2 du 13 au 15 décembre 1985. Sciencies pour la communication, 18. Berna, Peter Lang, 1987. p. 15-81.

. «Discourse production in oral comunication». En: QUASTHOFF, U. M. (ed.) Aspects of oral comunication. Research in text theory, 21. Berlín-Nueva York, W. de Gruyter, 1995. p.30-66.

LUSCHER, J. M. «Connecteurs et marques de pertinence. L'exemple d'Ailleurs». Cahiers de Linguistique Française 10, 1989. p.101-145.

MOESCHLER, J. et al. (ed.) Langage et pertinence: Référence temporelle, anaphore, connecteurs et métaphore, Processus discursifs - Langage et cognition, Nancy, Presses Universitaires de Nancy, 1994.

PAILLARD, D. «D'ailleurs ou comment enchaîner l'un a l'autre (Essai de traitement lexicologique». Le gré des langues 2, 1991. p.60-67.

ROULET, E. «Complétude interactive et connecteurs reformulatifs». Cahiers de Linguistique Française 8, 1987, p. 111-140.

SPERBER, D.; WILSON, D. (1986) La relevancia, Lingüística y conocimiento. Madrid, Visor, 1994.

VICTORRI, B. «La construction dynamique du sens». En: PORTE, M. (dir.) Passion des formes - Essais pour René Thom. Saint Claud, Éditions de l'ENS de Fontenay-Saint-Cloud, 1996. p. 733-747. 